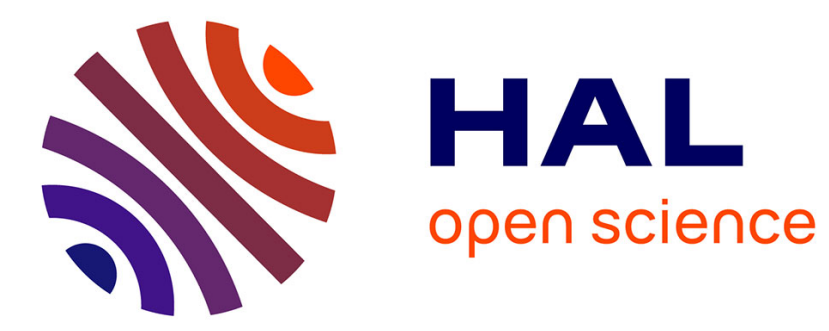

\title{
Compte rendu par les organisateurs
}

\author{
Laurent Debarbieux, A. Dublanchet, O. Patey
}

\section{To cite this version:}

Laurent Debarbieux, A. Dublanchet, O. Patey. Compte rendu par les organisateurs. Médecine et Maladies Infectieuses, 2008, 38 (8), pp.410 - 414. 10.1016/j.medmal.2008.06.018 . pasteur-03165438

\section{HAL Id: pasteur-03165438 \\ https://hal-pasteur.archives-ouvertes.fr/pasteur-03165438}

Submitted on 10 Mar 2021

HAL is a multi-disciplinary open access archive for the deposit and dissemination of scientific research documents, whether they are published or not. The documents may come from teaching and research institutions in France or abroad, or from public or private research centers.
L'archive ouverte pluridisciplinaire HAL, est destinée au dépôt et à la diffusion de documents scientifiques de niveau recherche, publiés ou non, émanant des établissements d'enseignement et de recherche français ou étrangers, des laboratoires publics ou privés.

\section{(이)(\$)}

Distributed under a Creative Commons Attribution - NonCommercial - NoDerivatives| 4.0 


\section{COMPTE RENDU par les Organisateurs \\ Laurent DEBARBIEUX, Alain DUBLANCHET \& Olivier PATEY}

\section{Session : Limites de l'Antibiothérapie \\ Intervenants : V. Jarlier, F. Trémolières}

Vincent Jarlier (laboratoire de microbiologie et Hygiène, CHU Pitié Salpêtrière) nous a tout d'abord rappelé la situation microbiologique à laquelle nous sommes confrontés aujourd'hui. De plus en plus de bactéries multirésistantes envahissent non seulement nos hôpitaux, mais également la ville tel Staphylococcus aureus (SA) méthicillino-résistant (SAMR), et diffusent ensuite rapidement après avoir acquis leurs gènes de résistance. Cette antibio-résistance varie selon les pays européens avec un gradient croissant du nord au sud et d'ouest en est. C'est ainsi que 35\% des SA sont résistants à la méthicilline. Le pourcentage d'entérobactéries productrices de bêta-lactamases à spectre étendu (BLSE) ne cesse de croitre avec une modification de leur répartition (augmentation des colibacilles et diminution des klebsielles et Enterobacter). Aujourd'hui, 3\% des E.coli isolées de bactériémies sont productrices de BLSE et $11 \%$ résistants à la ciprofloxacine. Les bonnes pratiques d'hygiène hospitalière (lavage des mains, utilisation des solutions hydroalcooliques...) ont permis une diminution importante des SARM. Enfin, un nombre croissant de staphylocoques à sensibilité diminuée aux glycopeptides et d'entérocoques résistants à la vancomycine sont observés.

Parallèlement, FrançoisTrémolière a rappelé la faible recherche en antibiothérapie avec l'absence d'innovation dans les familles d'antibiotiques. Il rappelait que les antibiotiques sont un bien durable à sauvegarder ; la dernière période « d'euphorie » remonte aux années 80-90. La grande efficacité de ces traitements souvent de courte durée, pour la plupart " génériqués », ne sont plus rentables pour les firmes pharmaceutiques. On guérit aujourd'hui une maladie mortelle comme la pneumonie à pneumocoques pour quelques euros ! Les antibiotiques anciens sont donc menacés. Un véritable Grenelle International de la valorisation et de la préservation des antibiotiques est nécessaire.

\section{Session Bactériophages et Phagothérapie}

Intervenants : S. Jacquet, A. Dublanchet, E. Fruciano

Avec Stéphan Jacquet (INRA, Thonon-les-Bains) les bactériophages ont été situés dans la nature. Ce chercheur qui étudie l'écologie microbienne des lacs alpins, a montré l'importance quantitative et fonctionnelle des virus en milieu aquatique. La présence environnementale est essentielle à bien des égards, montrant que les bactériophages sont les entités biologiques les plus abondantes et les plus diversifiées au sein des milieux aquatiques et que leur rôle est primordial en tant qu'agents de contrôle de la biomasse bactérienne, de la structuration des communautés microbiennes ou encore des grands cycles biogéochimiques.

Quelques chiffres et exemples montrent la place des bactériophages. Partout où il $\mathrm{y}$ a des bactéries, il y a des phages. Mis bout à bout, à la manière d'un collier de perles, les $10^{30}$ virus présents dans les océans mesureraient plusieurs centaines de milliers d'années lumières, suggérant que les eaux puissent être une ressource inépuisable de bactériophages. Il est admis aujourd'hui que les virus interviennent dans la structure, la composition et la mortalité des microorganismes aquatiques (procaryotes et eucaryotes), et sont par conséquent susceptibles d'influencer les flux de matière et d'énergie y compris de gènes entre les microorganismes. Jusqu'à $10^{23}$ infections virales ont lieu chaque seconde dans l'océan et une diversité insoupçonnée il y a encore quelques années, caractérise, cette communauté. Depuis la fin des années 1980, l'écologie virale est devenue une discipline à part entière et l'on sait aujourd'hui que l'on ne peut plus ignorer ces particules virales si l'on veut expliquer le fonctionnement des réseaux trophiques aquatiques ou encore l'incroyable diversité microbienne en leur sein.

Nous avons abordé la phagothérapie probablement-dite par l'histoire avec l'intervention d'Alain Dublanchet. Il nous a rappelé que c'est d'Herelle qui a relaté les toutes premières tentatives de phagothérapie chez cinq enfants atteints de dysenterie bacillaire à Hôpital Necker-Enfants 
Malades au cours de l'été 1919. Deux années auparavant, il avait fait la description in vitro du phénomène de lyse bactérienne et l'isolement du "microbe" bactériophage (Bacteriophagum intestinale) puis développé une théorie sur la guérison naturelle et l'extinction des épidémies. Toutefois on considère que les premiers essais thérapeutiques furent divulgués par Bruynoghe et Maisin en 1921. Dans les années qui suivirent, les publications scientifiques ou même de vulgarisation, les soutenances de thèses, furent très nombreuses. On en dénombre environ 150 par an, décrivant le traitement de pathologies diverses (voir article Dublanchet). On ne sera pas surpris qu'en l'absence d'autres moyens, et dans un contexte où les maladies infectieuses faisaient des ravages, cette thérapie antibactérienne souvent présentée comme "miraculeuse", eut un retentissement mondial. Plusieurs firmes pharmaceutiques proposèrent des suspensions utilisables dans diverses indications. Ainsi, bien avant 1939, la phagothérapie était répandue et installée dans le monde.

Toutefois alors que les demandes de traitements augmentaient, la production ne suivait pas ni en quantité, ni en qualité. Les problèmes étaient nombreux dont, en premier lieu, l'inconstance des résultats. Devant une telle variabilité le doute se répandit ... alors que l'antibiothérapie, à son tour, naissait.

Emiliano Fruciano (Écoles des Hautes Études en Sciences Sociales) a alors expliqué qu'à cela s'ajouta les théories de d'Hérelle, hétérodoxes pour l'époque, qui se heurtèrent à bon nombre de pasteuriens. En effet, d'Herelle croyait que le seul agent biologique bactériolytique était le bactériophage. Aux questions suivantes telles que : pourquoi certains patients guérissent-ils spontanément des maladies infectieuses d'origine bactérienne ? Pourquoi après un certain délai les épidémies s'éteignent? Quelle est la cause de ces phénomènes? Félix d'Herelle aurait sans aucun doute répondu : «C'est le bactériophage, agent contagieux de la guérison naturelle ». La phagothérapie n'est que l'application pratique de la théorie de la « guérison naturelle ». Soigner une maladie, en prévenir la propagation ou arrêter une épidémie signifie reproduire artificiellement les processus dans lesquelles en nature une maladie se termine ou une épidémie s'éteint. Plus encore, pour lui, les mécanismes de défense immunitaire - cellulaires et humoraux - n'étaient pas capables de défendre l'hôte parce qu'ils ne donnaient pas lieu à la production, dans un temps suffisamment court, de substances réellement bactériolytiques. Par conséquent, d'Herelle affirmait que la sérothérapie et la vaccination étaient inefficaces voire dangereuses. Selon lui, l'histoire de l'immunologie aurait même été l'histoire d'une erreur. Ainsi peut-on trouver une raison supplémentaire au destin singulier de la phagothérapie et de son promoteur, Félix d'Herelle dont la théorie à l'opposait l'Institut Pasteur et à la médecine institutionnelle.

\section{Session : Phagothérapie Expérimentale}

Intervenants : H. Brüssow, L. Debarbieux, P. Bifani, O. Grossi, A. Comeau

Impliqué depuis plusieurs années dans un projet de phagothérapie au sein du centre de recherche de Nestlé, Harald Brüssow débuta cette session par une longue communication axée sur l'utilisation de bactériophages du type T4 afin de lutter contre les diarrhées infantiles qui tuent chaque année des millions d'enfants dans les pays du tiers-monde.

Le bactériophage T4 est l'un des bactériophages modèles les mieux étudiés, faisant de lui le prototype des bactériophages lytiques. On peut lui donner le surnom de «tueur professionnel », par opposition au phage Lambda qui lui aussi est capable de lyser des bactéries la plupart du temps, mais qui, faisant partie de la famille des phages tempérés, possèdent des activités enzymatiques lui permettant d'insérer son matériel génétique dans le chromosome bactérien. De telles activités enzymatiques n'ont jamais été identifiées dans le génome du bactériophage T4, permettant à celui-ci d'être considérer comme inoffensif dans le remaniement des chromosomes bactériens.

Dans le but de lutter contre les diarrhées infantiles, l'équipe de H. Brüssow a été sur le terrain pour isoler des bactériophages à partir de selles de patients, afin d'obtenir des phages capables de lyser les souches responsables de ces diarrhées. Cette recherche s'est orientée sur l'isolement de bactériophages de type T4. Cela fût effectué en cultivant les différents bactériophages issus des selles sur la souche de laboratoire E.coli K-12. Cette souche ayant été complètement séquencée et étant totalement dépourvue de pouvoir pathogène pour l'humain, elle permet de réaliser des amplifications 
de bactériophages dans des conditions de sécurité maximale (les débris cellulaires ne contiennent pas de toxines potentielles). En comparant les spectres d'hôtes de chacun de ces phages il est apparu qu'une combinaison réduite de quelques-uns des phages T4 permettait de lyser in vitro plus de $80 \%$ des souches entérotoxinogénes et entéropathogénes d'E.coli.

Des essais sur animaux ont été entrepris afin d'identifier d'éventuels effets des bactériophages sur les bactéries commensales. Les bactériophages précédemment isolés ne sont pas spécifiques des bactéries pathogènes, ils sont aussi capables de lyser des E. coli commensales, puisqu'ils ont été propagés avec E.coli K-12. Donnés oralement à des souris à une concentration de $10^{3}$ à $10^{6} \mathrm{pfu} / \mathrm{ml}$, les bactériophages sont retrouvés dans les selles plusieurs heures plus tard, démontrant que les bactériophages peuvent traverser l'intestin sans perdre leur activité. Le suivi des bactéries commensales au cours du traitement ne fait apparaitre aucune différence significative suggérant que les bactériophages ne font que passer. Un examen approfondi de coupes de l'intestin et du colon révèle que les souches commensales d'E.coli localisées dans le colon résident dans le mucus, loin de la surface des cellules de l'épithélium intestinal. Cette observation soulève deux questions : d'une part comment les souches pathogènes arrivent à établir un contact avec les cellules épithéliales du petit intestin, et d'autre part est-ce que les phages donnés oralement ont la possibilité d'atteindre leur cible?

Les récentes études sur les bactériophages du genre T4 effectuées notamment par le groupe de H.M. Krisch ont permis de montrer que la protéine majeure de la capside était un bon marqueur de la phylogénie de cette famille de bactériophages. Le séquençage de différents nouveaux phages T4 a permis de compléter les arbres phylogénétiques de cette famille, et a permis de montrer que tous ces phages étaient regroupés dans la même branche suggérant qu'ils dérivaient d'un ancêtre commun. D'autre part, à l'aide d'informaticiens une base de données de gènes indésirables a été construite. Cette base contient tous les gènes identifiés codant pour des toxines et autres protéines toxiques ou impliquées dans la résistance aux antibiotiques. En confrontant cette base de données avec les séquences des génomes des nouveaux bactériophages T4 il ne fût pas détecté la moindre homologie significative confirmant une fois de plus que les bactériophages de la famille du phage T4 sont bien dépourvus de toute activité recombinante.

Enfin des essais cliniques de phase 1 ont été réalisés avec des volontaires. En donnant dans l'eau de boisson une quantité de $10^{5} \mathrm{pfu} / \mathrm{ml}$ du bactériophage T4, celui-ci est retrouvé dans les selles quelques heures plus tard. Ainsi les phages passent à travers l'estomac et les intestins sans perdre leur activité. Le suivi de l'impact de ces phages sur les bactéries commensales révèle qu'elles ne sont pas affectées par ce traitement. De plus, il ne fût noté aucun effet particulier pendant la durée de ce traitement. Aucune trace de phage ne fût retrouvée dans le sang de même qu'aucune réponse immunitaire ne fût détectée.

Pour terminer H. Brüssow aborda la question des phages de bactéries à Gram positif en prenant comme exemple les phages de la famille SPO1 qui sont l'équivalent de la famille T4. Eux aussi sont dépourvus de gènes leur permettant de recombiner leur matériel génétique et leur génome ne contient aucun gène « indésirable ». Pour illustrer son propos il prit comme exemple des prophages de Streptococcus pyogenes dont le génome a été séquencé. Ceux-ci possèdent clairement des séquences qui ne sont pas relatives à la physiologie du phage mais probablement issues de précédents évènements de recombinaison. De plus, il est maintenant prouvé que dans les génomes des bactéries pathogènes il existe un grand nombre de prophages et que ceux-ci sont souvent porteurs de gènes de virulence. Le record est peut-être même atteint avec le génome de E.coli O157 :H7 qui contient un si grand nombre de prophages que l'on pourrait presque dire que cette bactérie n'en n'est plus une, mais est devenue un énorme prophage.

En conclusion, il est clairement établi que l'utilisation de phages tempérés en phagothérapie est à écarter à cause de leur capacité à transmettre par recombinaison des gènes de toxicité, de virulence, voire de résistance aux antibiotiques. Il est donc nécessaire de se tourner vers des phages lytiques, «tueurs professionnels », qui n'ont toujours entretenu que des relations proie/prédateur.et n'ont jamais appris à co-évoluer avec leur hôte. 
Laurent Debarbieux s'est attaché à présenter un projet d'utilisation des bactériophages pour lutter contre les infections respiratoires. A l'aide d'un modèle d'infection pulmonaire par Pseudomonas aeruginosa chez la souris, il a démontré que les bactériophages pouvaient permettre la survie d'animaux exposés à des doses létales de bactéries. Ce modèle d'infection aigüe est réalisé par une application intra nasale des bactéries. L. Debarbieux a démontré qu'une dose équivalente de bactériophages administrée de la même manière deux heures après les bactéries était suffisante pour améliorer considérablement la survie, celle-ci étant totale lorsque l'on administre 10 fois plus de bactériophages que de bactéries. Enfin le suivi de l'évolution de l'infection et de son traitement a été effectué par l'utilisation d'une souche de $P$. aeruginosa luminescente. Une telle souche permet de suivre chaque animal grâce à un équipement qui permet de détecter la lumière émise à travers le corps de l'animal celui-ci étant légèrement endormi pendant le temps de la mesure. Enfin, les phages utilisés dans cette étude ont été nouvellement isolés de l'eau d'égout et amplifiée sur la souche infectieuse pour l'animal. Cependant certains de ces phages sont capables de lyser des souches isolées de patients suggérant que l'extrapolation (au moins du côté des phages) de l'efficacité d'un tel traitement n'est pas si éloignée.

Pablo Bifani effectua une brève introduction sur la tuberculose et les stratégies actuellement utilisées pour lutter contre la bactérie Mycobacterium tuberculosis. Le projet qu'il a présenté a pour but d'utiliser les bactériophages non pas pour soigner la tuberculose elle-même, mais pour diminuer son incidence en limitant sa transmission et en diminuant la durée du traitement, permettant de réduire aussi l'émergence de souches multirésistantes. Pour rappel, le traitement actuel dure 6 mois dont 3 avec 4 drogues et les 3 suivants avec 2 drogues. Or il a été montré que durant le premier mois de l'infection, $M$. tuberculosis est retrouvé en grand nombre dans les poumons des patients. Un traitement utilisant des bactériophages en aérosols pourrait ainsi réduire le nombre global de bactéries diminuant de ce fait les risques de contamination de l'entourage des patients.

A partir de différentes sources, un grand nombre de phages a été isolé et leur spectre d'hôte a été déterminé, conduisant à la caractérisation de phages ayant un large spectre d'hôte parmi les souches cliniques de $M$. tuberculosis. Ces phages ont ensuite été testés sur d'autres souches de Mycobacterium et la plupart se sont révélés inefficaces suggérant qu'il existe des différences assez importantes dans les protéines de surface des ces différentes souches, impliquant qu'il existe probablement une grande variété de bactériophages.

Parmi les phages isolés un seul est capable de lyser une série de souches représentatives des souches cliniques de $M$. tuberculosis. Ce phage de la famille des siphoviridae a été séquencé et son génome est en cours d'analyse. Ce phage présente la particularité d'avoir un point isoélectrique assez bas lui permettant d'interagir de manière électrostatique avec des petits peptides pénétrants. De tels peptides ont été identifiés comme capables de pénétrer les cellules eucaryotes. En ajoutant à une culture de cellules de macrophages infectées par M. tuberculosis, un mélange peptides plus phages on peut observer que les phages sont capables de pénétrer à l'intérieur des macrophages et de lyser $M$. tuberculosis. La prochaine étape sera de modifier le génome du phage pour qu'il puisse exprimer une protéine de la capside qui porte ce peptide en espérant que celui-ci garde ses propriétés pénétrantes. $M$. tuberculosis étant un pathogène intracellulaire cette modification est souhaitable sous peine de constater une inefficacité des phages.

L'utilisation d'un modèle murin a permis de montrer que le phage administré par voie intranasale possède une durée de vie très courte. Par contre administré par voie intra-trachéale sa durée de vie est allongée suggérant que le passage par voie nasale est délétère pour ce phage. Afin de contourner ce problème le phage a été exposé à une irradiation par UV afin de générer des variants à durée de vie allongée. De tels variants ont en effet été isolés et la demi-vie de ces phages est maintenant de plusieurs jours.

Les conclusions de ce travail renforcent la nécessité d'utiliser des phages lytiques, à durée de circulation étendue, non immunogène (les phages entiers sont rarement immunogènes, alors que les protéines isolées des phages le sont), et présentant une propriété de pénétration des cellules eucaryotes. Enfin P. Bifani mentionna que les souches de M. kansasis transformées par l'ADN du 
phage de M. tuberculosis peuvent permettre de produire des particules virales actives sans que les souches productrices ne soient lysées.

Olivier Grossi présenta une étude préliminaire sur le traitement expérimental d'une infection ostéo-articulaire à Staphyloccocus aureus chez le lapin. Les infections ostéo-articulaire à $S$.aureus représente 40 à $60 \%$ des cas d'arthrite aïgue ainsi qu'environ $30 \%$ des cas d'infections de prothèses. Ces infections sont difficiles à traiter à cause de multiples facteurs tels que la résistance aux antibiotiques, la formation de biofilms, la localisation intracellulaire des bactéries dans les cellules phagocytaires et les ostéoblastes, la réponse pro-inflammatoire de l'hôte par l'activation de l'IL-6 favorisant l'inflammation et inhibant l'apoptose...

Dans la littérature, plusieurs articles décrivent l'utilisation des bactériophages pour lutter contre des infections à $S$. aureus, alors qu'un nombre réduit d'articles relate l'utilisation des bactériophages dans des infections osseuses.

Le modèle développé par Grossi et ses collaborateurs est un modèle d'ostéomyélite subaigüe chez le lapin. La souche utilisée est une souche multirésistante aux antibiotiques isolée d'un patient, et la source de bactériophage est une suspension commerciale d'origine russe. Une suspension fraiche de bactériophages a été obtenue après le passage répété de la solution initiale sur la souche utilisée pour l'infection. Cette suspension a été utilisée pour tester sa capacité à lyser les bactéries dans un milieu complexe en utilisant des caillots de fibrine infectés. Dans ce modèle les phages se sont révélés actifs.

Dans le modèle d'ostéomyélite chez le lapin, 3 jours après le début de l'infection, les phages ont été appliqués pendant 2 jours, puis les animaux ont été sacrifiés. Les résultats montrent que les bactériophages sont inefficaces dans ce modèle. Plusieurs raisons peuvent être avancées comme l'inaccessibilité des phages aux bactéries intracellulaires, des conditions physico-chimiques non favorables à l'infection des phages, une durée de traitement trop courte. Plusieurs paramètres seront modifiés dans le futur afin de déterminer pour quelles raisons les bactériophages ne semblent pas efficaces dans ce modèle expérimental.

André Comeau exposa son travail récemment publié ${ }^{1}$ à propos de l'identification d'une synergie entre les phages et les antibiotiques. Cette découverte fût fortuite. Un médecin, examinant une boite Pétri sur laquelle avait été étalé directement un extrait de l'urine issu d'un patient, observa des plages de lyse dont certaines étaient plus larges autour des disques contenant des antibiotiques. Cette observation fût reproduite avec le phage et la souche isolée de cette première observation, mais aussi avec d'autres souches et d'autres phages. Cette augmentation de la taille des plaques de lyse traduit en fait soit une augmentation de nombre de phages produits, soit une augmentation de la rapidité de la lyse des bactéries. Cette synergie entre les phages et les antibiotiques a été identifiée avec différentes classes d'antibiotiques ( $\beta$-lactames, céphalosporines, quinolones, mitomycine C...). Elle n'est observée que pour des concentrations sub-létales d'antibiotiques. Ces faibles concentrations provoquent la filamentation des bactéries. Chez E.coli cette filamentation est sous le contrôle de la réponse SOS. Des mutants de cette voie de réponse au stress ont été utilisés pour montrer que la synergie phages-antibiotiques est indépendante de cette voie de signalisation. D'autre part des souches mutantes qui filamentent de manière constitutive ont permis de montrer que cette synergie était toujours présente.

Dans la perspective d'une utilisation thérapeutique des bactériophages, il est important de souligner que l'utilisation conjointe avec de faibles doses d'antibiotiques pourrait se révéler avantageuse pour les patients. D'un point de vue écologique, la mise en évidence de cette synergie bouscule un principe longtemps admis selon lequel tout ce qui favorise la croissance bactérienne favorise en retour la multiplication des bactériophages. D'autre part cette synergie n'étant pas observée avec tous les bactériophages elle pourrait être considérée comme un avantage dans un environnement riche en bactériophages. A. Comeau suggère que l'on pourrait parler de mutualisme

\footnotetext{
${ }^{1}$ Comeau, A. M., F. Tétart, et al. (2007). "Phage-Antibiotic Synergy (PAS): $\beta$-Lactam and Quinolone Antibiotics Stimulate Virulent Phage Growth "PLoS ONE 2(8). 
entre les cellules productrices d'antibiotiques et les bactériophages aboutissant à la destruction plus importante de compétiteurs.

\section{Session Phagothérapie Appliquée}

Intervenants : R. Adamia, M. Kutateladze, R. Rakin, M. Zizi

Les données récentes concernant l'utilisation de la phagothérapie en pathologie humaine restent peu nombreuses et proviennent principalement d'Europe de l'Est (Géorgie et Pologne). Il existe notamment à Tbilissi depuis 80 ans un centre de production de phages thérapeutiques, l'Institut Eliava. Les données issues de ce centre font l'objet d'un article de Mzia Kutateladze et Revaz Adamia dans le même numéro de ce journal. Aujourd'hui à Tbilisi (Géorgie), des firmes se sont séparées pour commercialiser des préparations. Ainsi, il est possible de trouver des produits fabriqués « artisanalement » mono-spécifiques (anti-staphylocoque, streptocoque, pyocyanique, colibacille..) et des produits polyphagiques (Intestinophage ${ }^{\mathrm{TM}}$ Pyophage $^{\mathrm{TM}}$, PhagoBioderm ${ }^{\mathrm{TM}}$ ). Ces phages sont utilisés en traitement curatif et préventif, soit seuls, soit associés à une antibiothérapie. En outre, signalons que très récemment (2006), une société américaine (Intralytix) et une sociéte hollandaise (EBI Food Safety) ont obtenu de la FDA la norme GRAS (Generally Recognised as Safe) et l'autorisation de commercialiser un produit anti-Listeria (respectivement LMP-102 ${ }^{\mathrm{TM}}$ et Listex ${ }^{\mathrm{TM}}$ ) dans l'industrie alimentaire (fromage, viande).

Deux présentations orales rapportaient l'utilisation de ces phages en Europe de l'OUEST, respectivement en Allemagne pour des cas ponctuels d'infections à SARM, bacille pyocyanique ou Proteus (pieds diabétique, brûlures, ostéite chronique) et en Belgique dans le cadre d'une étude contrôlée comparant bactériophages et traitement standard chez des grands brûlés. Dans les deux cas, les auteurs avaient obtenus l'autorisation d'un comité d'éthique pour l'utilisation d'un mélange de phages purifiés ayant fait l'objet de contrôles de qualité selon les normes européennes. En effet, à ce jour les suspensions de bactériophages à usage thérapeutique ne sont pas bien définies sur le plan juridique comme le seraient les antibiotiques ou des dispositifs médicaux, empêchant actuellement une AMM. Les protocoles thérapeutiques doivent donc faire l'objet d'un avis favorable d'un comité d'éthique.

L'équipe de Martin Zizi (Département de Physiologie, CHU de Bruxelles) a sélectionné un mélange de 3 phages actifs sur les souches habituellement isolées dans leur centre de grands brûlés, 2 vis-à-vis de bacilles pyocyaniques et un autre actif sur les SARM. Les auteurs ont rappelé les différences existant entre bactériophages et antibiotiques : les premiers sont très spécifiques, sans effet secondaire notable, faciles à produire mais nécessitant l'isolement préalable de la bactérie. Les seconds sont quand à eux à large spectre, avec de nombreux effets secondaires potentiels, peu spécifiques avec modifications de la flore endogène et la mise au point de nouveaux antibiotiques est difficile. Leur protocole thérapeutique n'a débuté qu'en septembre 2007 et les résultats ne sont donc que très préliminaires. L'évaluation a été faite selon les normes occidentales tant dans la préparation de la suspension phagique (BFC 1 contenant les 3 bactériophages) que dans la conduite de l'étude. Il s'agissait de comparer la bactériologie de surfaces brûlées contigües l'une traitée par une application par spray des phages, l'autre servant de contrôle. Sur les 4 patients traités, dans trois cas il n'y avait pas de différence entre les 2 zones cutanées, l'un par un traitement standard l'autre par les bactériophages ; dans le dernier cas il y avait une baisse significative de la charge bactérienne. Aucun effet secondaire n'était observé.

Les phages d'une préparation commerciale (D\&D Pharma GmbH, Allemagne) utilisés par Alexander Rakin et Nodar Daniela (respectivement de l'Institut d'Hygiène et de Microbiologie Médicale à l'Université de Munich et du département de traumatologie à Hanovre) sont dirigés sur les SARM (DePhag®) et sont sans effet sur la flore normale de l'hôte. Ils ont permis dans les 9 cas rapportés d'infections chroniques osseuses et des tissus mous l'éradication bactérienne avec la guérison clinique de l'infection. Les traitements classiques associant chirurgie et antibiothérapie n'avaient permis un tel résultat. Les frissonnements observés sont probablement attribuable à la libération d'endotoxine après la destruction d'un grand nombre de bactéries pathogènes. 
Enfin, signalons deux observations françaises, ponctuelles, qui ont été rapportées sous forme de communications affichées. Pour la première, il s'agissait d'une jeune adulte de 20 ans présentant une otite externe à SARM rebelle (durant 6 mois) à de multiples tentatives de traitement classique et qui a évoluée de manière spectaculaire (en quelques jours) sous phagothérapie locale (Alain Dublanchet, laboratoire de Microbiologie, CHI de Villeneuve Saint Georges). L'autre cas clinique concernait le traitement d'une infection pulmonaire à pyocyanique d'une femme médecin de 54 ans au passé infectieux très lourd. Les bactériophages ont été administrés par aérosol. Les résultats n'ont pas été immédiats mais ont permis une intervention sous antibiotiques et finalement l'éradication temporaire de la bactérie pathogène (Jean-Marie Duez, laboratoire de Bactériologie, CHU de Dijon).

Dans toutes ces observations, il s'agissait bien de phages naturels et non de phages manipulés génétiquement. 